\title{
Deutschsprachige Kurzprosa der Gegenwart an der Pädagogischen Fakultät: ein Unterrichtsvorschlag ${ }^{1}$
}

\section{Jan Budňák}

How to handle contemporary literature is not the competence that has been stressed in university teacher training in the Czech Republic. Such neglect means, however, losing a potent source of reading skill training as well as the students' and their future pupils' desire to read. The paper gives a possible course design for the contemporary short German fiction, aimed at students of German language at Faculties of Education. At the same time, it is the methodical resume of a FRVS project (FRVS/1356/2009).

contemporary fiction - short fiction - German as a foreign language - course design e-learning

Ein kompetenter Umgang mit Texten der deutschsprachigen Gegenwartsliteratur wird in der Hochschulausbildung von tschechischen Deutschlehrern in der Regel nicht besonders betont, vermutlich wegen der „Unübersichtlichkeit“ bzw. Vielfältigkeit des Stoffes. So wird aber ein Stoff beiseitegelassen, der nicht nur die Lesekompetenz erheblich steigern könnte, sondern auch die Leselust.

Im Beitrag wird ein Unterrichtsversuch zur deutschsprachigen Kurzprosa der Gegenwart für tschechische DaF-Studierende vorgestellt. Gleichzeitig stellt der vorliegende Beitrag die Zusammenfassung des methodisch-fachlichen Teils eines vom FRVŠ geförderten Projektes dar (FRVS/1356/2009).

Gegenwartsliteratur - Kurzprosa - DaF-Unterricht - Unterrichtsentwurf - E-Learning

Im Bereich der universitären Ausbildung von Fremdsprachenlehrern ist die Gegenwartsliteratur in der Fremdsprache in vieler Hinsicht ein Sonderfall. Um gleich bei dem wohl folgenschwersten Punkt zu beginnen: Sie muss Studierenden, die ihre Ausbildung an einer tschechischen Lehrerbildungsanstalt durchlaufen, wo oft genug nur wohl Geordnetes vermittelt und gelernt wird, als der Inbegriff von Unüberschaubarkeit erscheinen. Gegenwartsliteratur stellt Studierende vor die Aufgabe, einen eigenen Weg durch einen erzählenden Text zu suchen, der nicht „deduktiv“ von der Epochenbeschreibung abgeleitet werden kann, wie das etwa bei einem Barockgedicht fast immer der Fall ist. „Die Tränen des Vaterlandes Anno 1636“ - oder auch Eichs „Inventur“ - werden allzu oft zu einer bloßen Bestätigung der bekannten Stichwörter Vanitas oder Kahlschlag heruntergespielt und dadurch eigentlich nicht durch Lektüre „erworben“, sondern nach dem passenden Muster ausgelegt. Im Gegensatz dazu ist der (fremdsprachige) Leser einem Text der Gegenwartsliteratur ausgeliefert.

1 Der Beitrag ist im Rahmen des vom FRVS (Fond rozvoje vysokých škol) geförderten Projektes FRVS/1356/2009 „Contemporary Short German Fiction“ entstanden. 
Mit neueren Texten kann also die Reduzierung auf das Epochen- oder Verfassertypische nur beschränkt vorgenommen werden. Die Überwindung der Unüberschaubarkeit ist also zugleich die Chance, die inhaltlichen, stilistischen oder etwa landeskundlichen Schwerpunkte nach eigenem Ermessen oder in Gruppenarbeit zu setzen, den Text darauf abzuklopfen, was er eigentlich enthält bzw. was für Fragen er selbst stellt, ohne dabei gleich auf das rein Illustrative umzusteigen. Weitere Gründe für die Beschäftigung mit Gegenwartsliteratur im Unterricht zukünftiger Deutschlehrer liegen großteils auf der Hand. Nicht nur aktuelle (oder aber verdrängte) politische oder landeskundliche Themen werden in der neuesten Belletristik behandelt. Darüber hinaus muntern die Texte durch die der Literatur eigene Perspektivierung die Studierenden dazu auf, sich in einen bestimmten Standpunkt emotional hineinzuversetzen und ihn dadurch zu „erleben“.

Die Lektüre der neuesten Belletristik bereitet den DaF-Studierenden aber oft auch recht große Schwierigkeiten. Im Gegensatz zu der anregenden „Kopfidentität“ ist die oft betonte Attraktivität der Texte aufgrund der zeitlichen Nähe problematisch, denn neueste Prosatexte sind oft von einer erstaunlichen Vielfalt von Stilen und Stilisierungen geprägt. Ein ungeübter Leser kann mit dem in Andeutungen oder Anspielungen sprechenden Text kaum umgehen. Die mangelnde Leseerfahrung lässt einfach die meisten Komponenten des Textes links liegen und der Text - und damit „Literatur“ als solche - verliert an Reiz. Das wäre bei zukünftigen Lehrern natürlich nicht die ideale Entwicklung. Kurz: Die Gegenwartsliteratur ist - auch für tschechische DaF-Studierende - ein Sprungbrett ins anregende Unbekannte.

Wir stehen also vor der Frage, wie die deutschsprachige Gegenwartsliteratur angehenden Lehrern vermittelt werden kann, die in der Regel kaum Erfahrung mit dem Lesen zusammenhängender Texte haben und eine eigene, nicht vorbearbeitete Lektüre oft nicht bewältigen können. Ich werde im vorliegenden Text versuchen, eine kurze Erzählung vorzustellen und einige Tipps zur Behandlung von solchen Texten im Unterricht zu geben. Als Beispiel kann hier die kurze Erzählung „Alte Pralinen“ von der unlängst verstorbenen Autorin Undine Gruenter (1952-2002) dienen. Die Erzählung stammt aus dem posthum erschienenen Band Sommergäste in Trouville (2003). Der nur etwa 10 Seiten lange Text öffnet mit einem sehr einprägsamen Bild: „In einer kleinen Stadt, in der die Hälfte der Häuser leersteht, sind Gerüchte wie alte Pralinen, die im kühlen Keller in einem Regal gestapelt werden und schon von einer pelzigen Schicht weißen Schimmels überzogen sind, da der Deckel der Schachtel nicht luftdicht schließt“ (GRUENTER: 2003, 109). Dann wird die Geschichte des dicklichen, nach außen hin sehr pflichtbewussten, aber emotional vernachlässigten Mädchens Aurelie erzählt. Aurelie kommt als fünfähriges Pflegekind zu Madame Haussier, die kurz nach der Hochzeit ihren Ehemann verloren hat und nun allein mit der Pflegetochter Aurelie lebt.

Die Erzählung enthält einige Punkte, auf die durch „Fragen zum Text“ (Organisatorisches dazu später) hingewiesen werden sollte. Ziel der Fragen ist 
einerseits das Textverständnis zu fördern und andererseits auf literarische Tendenzen aufmerksam zu machen, die den Text in eine gewisse literaturhistorische Nachbarschaft stellen, hier die der Gender-Literatur. Zunächst muss die Aufmerksamkeit auf die Erzählsituation gelenkt werden. Die meisten Passagen sind so inszeniert, dass verschiedene Gespräche von Madame Haussier mit ihren kleinstädtischen weiblichen Bekannten wiedergegeben werden, in denen über Aurelie gesprochen wird. Bis auf vereinzelte sachlich gehaltene Beschreibungen von Aurelies Handlungen sind sie die dominierende Quelle für die Erschließung der Figur Aurelie. Aurelie lernen die Leser eben nur durch die Augen von Madame Haussier kennen: Alles andere sind Vermutungen des Erzählers.

Selbst da, wo über Madame Haussier gesprochen wird und zunächst keine Figurenperspektive bemerkbar ist, lässt sich eben diese im Nachhinein erkennen: „Madame Haussier war eine gute Frau. Sie arbeitete in der Küche des Restaurants Les Mouettes und möblierte ihr einsames Leben mit einer Pflegetochter, Aurelie, die sie aufs Gymnasium schickte" (ebd.). Im Laufe des Textes kann bei aufmerksamer Lektüre festgestellt werden, dass Madame Haussier eben keine "gute Frau ist“, zumindest in Bezug auf Aurelie. Höchstens sie selbst bzw. manche ihrer Freundinnen würden sie so bezeichnen; und dies tun sie eben in der Erzählung. Das Bild, das sie von Aurelie verbreitet, entspricht also nicht der Wirklichkeit. Der folgende Passus z. B. (Aurelie wird vermisst) wird nach dieser Feststellung als Aussage über Aurelie unbrauchbar; wichtig ist er aber wohl als Beweis der Leistung des kleinstädtischen Ondits: „Sie erklärte Aurelies Verschwinden allen, mit denen sie sprach, als neueres Symptom aus der Serie Hospitalismus und seine Folgen und sah sie schon in eine Besserungsanstalt eingeliefert, weil sie auf der Flucht in einem Bauernhof eine Kanne Milch gestohlen hatte" (GRUENTER: 2003, 116). Der verschlossenen Aurelie wird hier etwas vorgeworfen, was nur in der Phantasie ihrer Pflegemutter existiert. Der Leser hat aber kaum die Möglichkeit, eine "Gegenstimme“ oder auch nur eine objektive Stimme zu vernehmen. Der Leser muss also hinter die „Voreingenommenheit“ des scheinbar nur Tatsachen wiedergebenden Erzählers kommen. Erst dann kann er sich die hier entscheidende Frage stellen, nämlich die Frage, was er tatsächlich über Aurelie weiß.

Die Erzählung läuft also schließlich auf die Kritik der „kleinen Bosheiten“ hinaus, die sich Manche erlauben, wenn sie sich in einer Machtstellung gegenüber Schwächeren wähnen. Madame Haussiers „unwichtige“, „kleine“ Nachreden, durch die sie ihr Ansehen in der Gemeinde auf Kosten der Pflegetochter aufrechterhalten möchte, schlagen um in die Zerstörung des Kindes. Für die Leser - in diesem Fall also die Studierenden - ist diese Schlussfolgerung umso wertvoller, als sie sie selbst haben ableiten - also erleben - müssen. Ohne aber die entfremdende Perspektivierung der Erzählsituation zu durchschauen, ohne also die Erzählstimme als die Stimme des Gerüchts erkannt zu haben, würde der Text unverständlich bleiben. 
Die didaktische Grundfrage ist hier, wie man einen DaF-Studierenden, der den Begriff „Erzählperspektive“ nicht kennt bzw. nicht analytisch anwenden kann, auf den Gedanken bringen kann, dass er das, was er liest, auch noch hinterfragen soll. Ohne die Feststellung, dass er eine bereits eingetönte Version der Wahrheit liest, ist aber kein Verständnis der Erzählung möglich. Zum Glück zeigt sich gerade in diesem Punkt der Vorzug der Gattung „Erzählung“. Der kurze Text enthält meist ein paar Signale, die bei aufmerksamer Lektüre den ganzen Text entschlüsseln und „öffnen“. In unserem Falle finden sich diese Stellen bezeichnenderweise gleich am Beginn und dann wieder am Ende der Erzählung. Das Einstiegsbild von kleinstädtischen Gerüchten, die wie schimmelige alte Pralinen sind und im Keller gestapelt werden, wurde oben schon zitiert. Auch der letzte Absatz des Textes hat dieselben Gerüchte zum Thema: „Es heißt, im Keller eines kleinen Hauses in der Rue des Roses in Trouville sitzt ein kleines Mädchen zwischen leer gegessenen Pralinenschachteln und blinzelt träge in die funzlige Birne“ (GRUENTER: 2003, 117). Der Kursleiter hat hier also die Aufgabe, auf diese scheinbar kleinen Dinge aufmerksam zu machen: dass alte Pralinen im Titel der Erzählung erwähnt werden, dass Aurelie sich - und ihre Katze - aus „,irgendeinem Grund“ fast nur von diesen Pralinen ernährt, dass Pralinen zu Beginn der Geschichte als ein zunächst ziemlich undurchschaubares Bild für kleinstädtische Gerüchte angeführt werden, dass der letzte Absatz mit „Es heißt“ beginnt und eine gruselig unbewegliche Kellerszene schildert, in der das schweigsame Mädchen - vielleicht, „man sagt das“ doch nur ganze Schachteln von Pralinen aufgegessen hat usf. Alle diese Szenen stellen nämlich die allzu augenfällige Deutung der Erzählung in Frage, die für unaufmerksame Leser bereits die Endstation ist: Aurelie war vom Pflegeheim so verstört, dass sie sich auch bei der „guten“ Madame Haussier nicht einleben konnte und schließlich von zu Hause flüchtet. Diese Interpretation würde nämlich auf der Stimme der Gerüchte beruhen.

Wie kann also mit Kurzprosa der Gegenwart im Unterricht umgegangen werden? Die Grundvoraussetzung einer sinnvollen Beschäftigung damit ist die Lektüre des Textes. Da genügen kein Auszug und keine Inhaltsangabe. Nur eine gründliche vorangehende Auseinandersetzung von allen Kursteilnehmern mit dem Text kann dem Ziel des Kurses, eine musterfreie Deutung in Gruppendiskussion zu erarbeiten, gerecht werden. Das ist die erste Funktion, die der begleitende E-Learning-Kurs erfüllen kann. ${ }^{1}$ Die vom Kursleiter ausgewählten Primärtexte können eingescannt und in der E-Learning-Umgebung, etwa als pdf-Dateien gespeichert werden. Diese können dann von den angemeldeten Studierenden mühelos und ohne Organisationsschwierigkeiten heruntergeladen und gelesen werden. Alle anderen

1 An der Pädagogischen Fakultät der Masaryk-Universität in Brno hat sich die Lernplattform Moodle bestens bewährt. 
Optionen für den Grundaufbau des Kurses als die Lektüre von allen Texten durch alle Kursteilnehmer erweist sich als weniger günstig, weil bedeutend weniger interaktiv.

Eine ungezielte, ungeregelte, kontextlose Lektüre wäre natürlich in vielen Fällen kontraproduktiv. DaF-Studierende an tschechischen Pädagogischen Fakultäten verfügen in der Regel nicht über die Kompetenz bzw. Motivation, einem literarischen Text seine weniger auffälligen Dimensionen abzugewinnen. Bei nicht erarbeiteter Lektüre könnte es zu schweren Missdeutungen kommen, meistens auf Grund von Überinterpretationen in einem bestimmten Bereich und Auslassungen in allen anderen. Dann entsteht der äußerst verbreitete und dennoch falsche Eindruck, dass Literatur eine Art Palaver ist, die all das tatsächlich „enthält“, was sie in jedem einzelnen Leser hervorruft. Daher könne sie auch nicht "gedeutet“ werden, wird immer wieder angemerkt. Freilich hat diese Ansicht eine gewisse Berechtigung, aber sie kann nicht von einem DaF-Studierenden, sondern höchstens von einem Kafka-Forscher geäußert werden. Wie kann also ein ausgeglichenes und mehrseitiges Textverständnis angebahnt werden? Als eine gute Möglichkeit bieten sich lektürebegleitende Fragen zum Text an, die der Kursleiter ebenfalls im E-Learning-Kurs speichern kann. Die Formulierung der Fragen muss freilich davon abhängen, was die Leser auch ohne sie verstehen würden. In der Regel gilt, dass inhalts- und autorbezogene Fragen nicht unbedingt notwendig sind, da dies immer diejenigen Ebenen zu sein scheinen, wo fast kein Leser „Leerstellen“ in der Deutung des Textes toleriert. Entsprechende Informationen werden deshalb erfahrungsgemäß auch ohne Anleitung eingeholt. Interessanter - und komischerweise seltener gestellt werden „Warum“-Fragen und Fragen nach psychischen Vorgängen im Inneren einer Figur oder nach der ethischen Dimension der Figur bzw. der Handlung. Falls nach dem Urteil des Kursleiters die Studierenden im Stande sind, schreibtechnische bzw. ästhetische Merkmale des Textes zu reflektieren und den Begriffsschatz zu deren Besprechung zumindest verstehen können, können auch darauf hinauslaufende Impulse formuliert werden.

An dem Beispieltext können wir uns einige lektürebegleitende Fragen vergegenwärtigen. In erster Linie betreffen sie das bereits angedeutete Thema der „Parteilichkeit“ des Erzählers. Das Ziel der Fragen ist daher, die zentrale Metapher (Alte Pralinen $=$ Gerüchte in der Kleinstadt) in die Textinterpretation zu projizieren. Je textnäher die Fragen sind, desto besser. Hier seien zwei Beispiele gegeben: „Ist Madame Haussier wirklich ,eine gute Frau', wie es gleich auf der ersten Seite der Erzählung von ihr heißt? Wie ist eigentlich das Verhältnis zwischen Madame H. und Aurelie? Was genau wissen wir von Aurelie, das uns nicht Madame Haussier sagt?“ Oder: „Überlegen Sie sich die möglichen Bedeutungsfacetten des Vergleichs zwischen alten Pralinen und Gerüchten, der gleich im ersten Absatz vorgestellt wird (GRUENTER: 2003, 109). Je mehr, desto besser. In welcher Form zeigen sich ,Gerüchte‘ auch im allerletzten Absatz des Textes?“ 
Die „Fragen zum Text“ sollten, wie schon erwähnt, noch zumindest ein zweites Ziel erreichen, und zwar eine Sensibilisierung der Studierenden für Tendenzen der Gegenwartsliteratur, die im Text konkrete Gestalt gewinnen. ${ }^{1}$ Ich halte es nicht für sehr sinnvoll, die Texte völlig zu „schubladisieren“, d. h. sie ganz zu „Programmschriften“ einer Gruppe von Autoren zu machen, wie das neuerdings oft etwa den Autoren aus der ehemaligen DDR geschieht. Im erwähnten Beispieltext „Alte Pralinen“" von Undine Gruenter lässt sich allerdings ein zentrales Merkmal festmachen, das ihn mit dem Schreiben von vielen anderen gegenwärtigen Autorinnen verbindet, nämlich dass der (weibliche) Körper zum Austragungsort von allerlei psychischen, sozialen oder sogar ideologischen (Christa Wolf) Konflikten gemacht wird. Bei der Textanalyse im Unterricht gilt es also, zuerst die konkrete Gestaltung dieser Übertragung zu untersuchen und dann auf das Typische dieses Verfahrens hinzuweisen. Diesbezüglich können etwa lektürebegleitende Fragen wie folgt gestellt werden: „Sammeln Sie aus dem Text Informationen über Aurelies Äußeres. Wie entwickelt sich ihr Körper im Laufe ihres Lebens? Worauf ist die Entwicklung zurückzuführen?“ Oder: „Wie spiegelt sich Aurelies Rolle in der kleinstädtischen Gesellschaft an ihrem Körper wider?“ Oder: „Sammeln Sie in der Erzählung Informationen über die Pralinen. Warum werden sie von den Einwohnern von Trouville so massenhaft eingelagert? Welche der Informationen, die Sie gefunden haben, machen sie noch den Gerüchten gleich?" Die Diskussion, die sich aus diesen Fragen ergeben sollte, ist dann auf die Entsprechung zwischen Aurelies Körper und ihrer Position in der Dorfgemeinschaft gelenkt worden. Erst nach dieser textanalytischen Unterhaltung kann m. E. die Information über das Bezugssystem „Körper“ in der gegenwärtigen gender-sensitiven oder feministischen Literatur richtig wirksam werden. Erst hier sollten Beispiele von außerhalb des analysierten Textes kommen, sei es Elfriede Jelineks traumatisierte „Klavierspielerin“ oder Christa Wolfs in körperliches Leiden umschlagender ideologischer Kampf im Roman „Leibhaftig“.

Im Weiteren kann eine erfolgreiche Auseinandersetzung mit einem unbekannten Text der Gegenwartsliteratur durch das Recherchieren nach Buchrezensionen gefördert werden. Diese Internetquelle ist - abgesehen von literarischen Zeitschriften, die nur im Ausland vorhanden und damit für Studierende an tschechischen Hochschulen kaum erreichbar sind - wohl die ergiebigste, was die „Sekundärquellen“ zur Kurzprosa der Gegenwart angeht. Es gibt zwar auch schon gedruckte „Literaturgeschichten“ zu den neuesten literarischen Tendenzen (z. B. BÖTTIGER: 2004), sie veralten aber verständlicherweise schnell und lassen sich aufgrund des doch begrenzten Umfangs nicht als „Fundgruben“ von Anregungen für die Lektüre und die Diskussion verwenden. Im Vergleich zu den gedruckten Quellen nutzt im Unterricht etwa die Internet-Sammlung von Rezensionen und Rezensionsnotizen

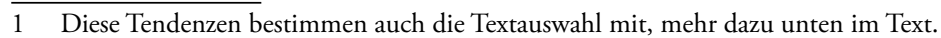


bei www.perlentaucher.de viel mehr. Eigentlich sind Rezensionsnotizen, so wie sie z. B. bei www.perlentaucher.de fast zu jeder Veröffentlichung zu finden sind, für die Studierenden gerade die richtige Gattung, wenn sie sich einen Überblick über das jeweilige Buch verschaffen wollen, samt den darin behandelten Themen, literarischen Darstellungstechniken und der Reaktion in den Medien darauf. Außerdem ist eine Rezensionsnotiz knapp und deutlich genug, um selbst auf leicht zu Ermüdende nicht ermüdend zu wirken. Ein weiterer Vorteil von Rezensionen besteht darin, dass sie sich meistens zu dem ganzen Erzählband äußern, und so bei der Besprechung einer einzelnen Erzählung Aufmerksamkeit auf Allgemeineres lenken, das oft zu kurz kommt: thematische Schwerpunkte, stilistische Eigenheiten, Gemeinsamkeiten in Figurenzeichnung usf. Im Unterricht hat sich die Variante bewährt, in der die Recherche von jeweils einem Kursteilnehmer durchgeführt und in der von ihr/ihm geleiteten Diskussion verwertet wird. Voraussetzung ist dabei die Lektüre des Textes durch alle.

Hier eine im Unterricht besprochene Rezensionsnotiz mit Fragen als Beispiel:

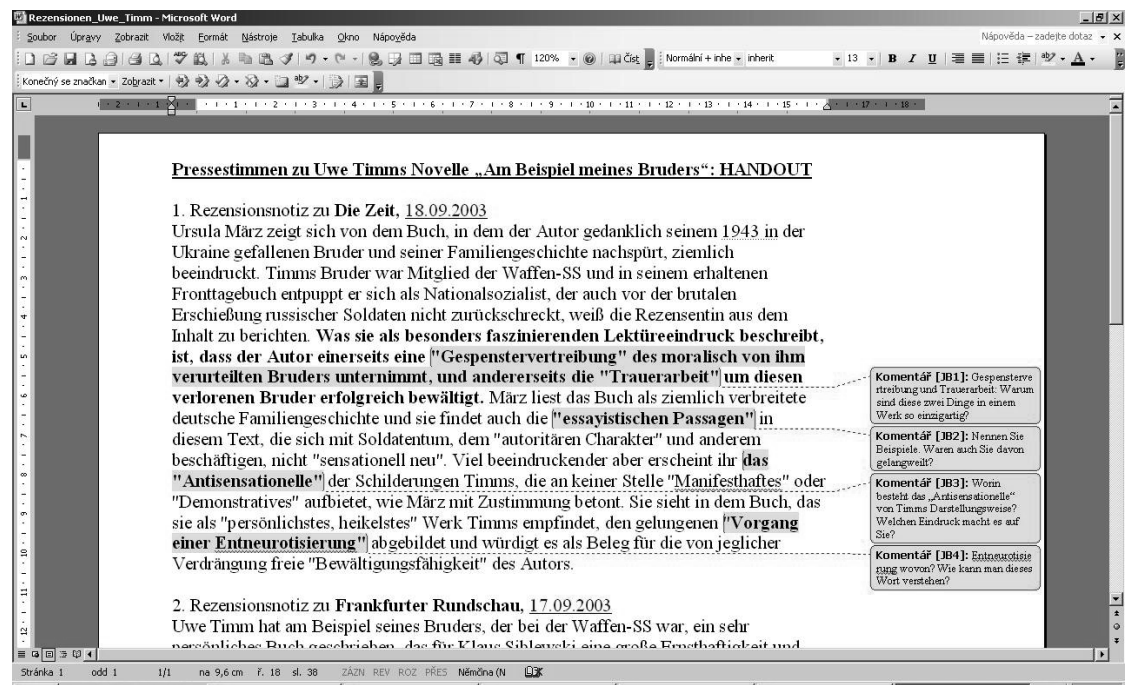

Eine kaum zufriedenstellend zu lösende Frage ist die Auswahl der Texte. Im Rahmen eines Seminars können jeweils höchstens 10 bis 12 Texte besprochen werden, ohne die Lesebereitschaft der tschechischen DaF-Studierenden zu überfordern. Obwohl das Kriterium „repräsentativ“ für die Textauswahl nicht unbedingt das Wichtigste ist, kommt den Studierenden ein "tendenzieller“ Charakter des Textes gelegen, spätestens bei der Bachelor- oder Masterprüfung, wo die Frage „Gegenwartsliteratur“ aufgrund ihrer Unüberschaubarkeit zu den meistgefürchtetsten gehört. 
Welche Texte sind a) den tschechischen DaF-Studierenden zugänglich und entsprechen b) einer bestimmten Tendenz der deutschsprachigen Gegenwartsliteratur? Abschließend soll hier eine kurze Liste von Begriffen für literarische Tendenzen der deutschsprachigen Literatur präsentiert werden, die in der öffentlichen Diskussion und in populärwissenschaftlichen Zeitschriften häufig fallen:

- „Vergangenheitsaufarbeitung“ (Uwe Timm: Am Beispiel meines Bruders),

- „Exilerfahrung“ (W. G. Sebald: Die Ausgewanderten),

- „Literatur von Autoren aus der DDR“ (Ingo Schulze: Handy),

- „Gender“, „weibliches Schreiben (?)“(Undine Gruenter: Alte Pralinen, Jenny Erpenbeck: Tand),

- „Multikulturelle Erfahrung“ (Feridun Zaimoglu: Zwölf Gramm Glück),

- „Postmodernes Schreiben“ (Bernard Schlink: Liebesfluchten, Daniel Kehlmann: Ruhm),

- „Generation Berlin“, „Generation Single“ (Judith Hermann: Nichts als Gespenster, Arno Geiger: Anna nicht vergessen),

- „Popliteratur“ (Christian Kracht: Mesopotamia).

Diese Erzählbände wurden von tschechischen DaF-Studierenden am Lehrstuhl für Deutsch der Pädagogischen Fakultät der Masaryk-Universität am positivsten aufgenommen und als Verdeutlichung der Entwicklungstendenzen der gegenwärtigen Literatur akzeptiert. Das wertvollste ist aber nicht die literaturgeschichtliche Information, sondern die Lektüreerfahrung (in der Fremdsprache), die zur Erweiterung der Lesekompetenz und persönlicher Bereicherung beiträgt - und nicht zuletzt zur Anbahnung einer entsprechend offenen Einstellung zum Lesen von Belletristik in der Fremdsprache an den Schulen führt.

\section{Literatur:}

BÖTTIGER (2004): Böttiger, Helmut. Nach den Utopien. Eine Geschichte der deutschsprachigen Gegenwartsliteratur. Wien: Paul Zsolnay, 2004.

GRUENTER (2003): Gruenter, Undine. Sommergäste in Trouville. Erzählungen. München: Carl Hanser, 2003.

TIMM (2003): Timm, Uwe. Am Beispiel meines Bruders. Köln: Kiepenheuer und Witsch, 2003.

http://www.perlentaucher.de/buch/15054.html, abgerufen am 30. September 2009;

http://moodlinka.ped.muni.cz/course/view.php?id=1554, abgerufen am 30. September 2009. 